\title{
Therapie und Prävention von Infektionskrankheiten
}

\section{Bibliografie}

Dol http://dx.doi.org/ 10.1055/s-0033-1358042

Drug Res 2014; 64, Suppl. 1: S1

(c) Georg Thieme Verlag KG

Stuttgart · New York .

ISSN 0004-4172

\section{Korrespondenzadresse Norbert Suttorp \\ Charité Universitätsmedizin Berlin \\ Charitéplatz 1 \\ 10117 Berlin \\ norbert.suttorp@charite.de}

\section{Stefan Endres}

Ludwig-Maximilians-Universität

München

Ziemssenstraße 1

80336 München

stefan.endres@lmu.de
Die Eindämmung von Infektionskrankheiten durch Hygiene, Gabe von Antiinfektiva sowie durch Vakzinierung war in den letzten 150 Jahren überaus erfolgreich. Diese Maßnahmen haben wesentlich zum Erreichen der jetzigen hohen Lebenserwartung und entscheidend zum Erreichen unserer beachtlichen Lebensqualität beigetragen. Infektionskrankheiten sind jedoch ein „moving target“, und frühere Erfolge in Therapie und Prävention sind, wenn wir nicht immer wieder adäquat reagieren, gefährdet. Mit Blick auf die sich derzeit in Ländern Westafrikas ausbreitende Ebolafieber-Epidemie hat dies dramatische Aktualität.

Die aktuellen Gefährdungen sind leicht benannt:

- drohender Verlust der Antibiotika-Wirksamkeit durch Ausbildung und Verbreitung von Resistenzen

- nicht ausreichende Wirksamkeit der Antiinfektiva bei schweren Infektionen

- globale Herausforderungen durch neue oder erneut auftretende Infektionskrankheiten

- sowie fehlende Vakzinen.

So steigt die Zahl der Fälle, in denen die verfügbaren Antibiotika nicht mehr wirken, und Organisationen und Institutionen wie die Weltgesundheitsorganisation, der G 8-Gipfel und der Europäische Ministerrat haben bereits mehrfach auf die Bedrohung durch Antibiotika-Resistenzen hingewiesen. In Deutschland legten die Nationale Akademie der Wissenschaften Leopoldina und die Akademie der Wissenschaften in Hamburg Anfang 2013 in ihrer Stellungnahme „AntibiotikaForschung: Probleme und Perspektiven“ eine umfassende Analyse einschließlich möglicher Gegenmaßnahmen vor.

Das Symposium wird in einer aktuellen Bestandsaufnahme insbesondere die folgenden Fragen adressieren:
- Was sind die aktuellen Entwicklungen bei Resistenzen gegen Antibiotika und wie können entsprechende Gegenstrategien aussehen?

- Welche adjunktiven (immunologischen) Therapiemöglichkeiten bei schweren, durch Bakterien, Viren und Pilze ausgelösten Infektionen, die über die Gabe von Antiinfektiva hinausgehen, sind in der Entwicklung?

- Welche neuen Behandlungsmöglichkeiten gibt es für Tuberkulose und Malaria, welche Strategien zur Prävention von Influenza- und Pneumokokken-Infektionen?

In der Tradition der Paul-Martini-Stiftung wird das Symposium etablierte Präventions- und Therapiestandards mit einem Ausblick auf die Forschungsaktivitäten von Universitäten, außeruniversitären Forschungseinrichtungen und forschenden Pharma-Unternehmen verbinden.

Traditionell bietet die Paul-Martini-Stiftung ein einzigartiges Forum, um Vertreterinnen und Vertreter dieser Bereiche zur Diskussion zusammenzubringen. Das Symposium soll daher besonders zum Diskurs zwischen universitärer und industrieller Forschung beitragen und weitere Impulse für innovative Therapiekonzepte geben.

Besonderer Dank gilt an erster Stelle allen Referenten, aber auch dem Vorstand der Paul-Martini-Stiftung und der Geschäftsstelle. Zusätzlich danken wir der Deutschen Akademie der Naturforscher Leopoldina, unter deren Mitwirkung und Schirmherrschaft dieses Symposium ausgerichtet wurde.

Norbert Suttorp

Charité Universitätsmedizin Berlin

Stefan Endres

Ludwig-Maximilians-Universität München 